Draft version September 13, 2018

Preprint typeset using $\mathrm{IAT}_{\mathrm{E}} \mathrm{X}$ style emulateapj v. 5/2/11

\title{
SCALING OF THE ELECTRON DISSIPATION RANGE OF SOLAR WIND TURBULENCE
}

\author{
F. Sahraoui ${ }^{1}$, S. Y. Huang ${ }^{2}$, J. De Patoul ${ }^{1}$, G. Belmont ${ }^{1}$, M. L. Goldstein ${ }^{3}$, A. Rétino ${ }^{1}$, P. Robert ${ }^{1}$, N. \\ CORNILlEAU-WEHRLIN ${ }^{1}$ \\ ${ }^{1}$ Laboratoire de Physique des Plasmas, CNRS-Ecole Polytechnique-UPMC, Route de Saclay, 92120 Palaiseau, France \\ ${ }^{2}$ School of Electronics and Information, Wuhan University, Wuhan, China and \\ ${ }^{3}$ NASA Goddard Space Flight Center, Code 673, Greenbelt 20771, Maryland, USA \\ Draft version September 13, 2018
}

\begin{abstract}
Electron scale solar wind turbulence has attracted great interest in recent years. Clear evidences have been given from the Cluster data that turbulence is not fully dissipated near the proton scale but continues cascading down to the electron scales. However, the scaling of the energy spectra as well as the nature of the plasma modes involved at those small scales are still not fully determined. Here we survey 10 years of the Cluster search-coil magnetometer (SCM) waveforms measured in the solar wind and perform a statistical study of the magnetic energy spectra in the frequency range $[1,180] \mathrm{Hz}$. We show that a large fraction of the spectra exhibit clear breakpoints near the electron gyroscale $\rho_{e}$, followed by steeper power-law like spectra. We show that the scaling below the electron breakpoint cannot be determined unambiguously due to instrumental limitations that will be discussed in detail. We compare our results to those reported in other studies and discuss their implication on the physical mechanisms and the theoretical modeling of energy dissipation in the SW.
\end{abstract}

Subject headings: turbulence, solar wind, heating, whistler, KAW

\section{INTRODUCTION}

The Solar Wind (SW) is certainly the astrophysical plasma in which significant progress in understanding plasma turbulence has been most achieved. The main reason for that is the availability of high quality data from several space missions, from the earlier spacecraft Voyager 1 and 2 to more recent ones such as Cluster, Wind or Stereo. While earlier spacecraft data have allowed for addressing turbulence at scales larger than the ion scale (typically $\rho_{i} \sim 100 \mathrm{~km}$, which corresponds to an observed frequency in the spacecraft frame of $f_{s c} \sim 0.5$ $\mathrm{Hz}$ using the Taylor assumption), i.e. the so-called inertial range, the high time resolution Cluster data have recently opened a new chapter in turbulence studies that focuses on electron scales $\left(L \sim \rho_{e} \sim 1 \mathrm{~km}\right)$ (Sahraoui et al. 2009; Kiyani et al. 2009, Alexandrova et al. 2009 Sahraoui et al. 2010a). These observations have driven intensive research work on electron scale turbulence both theoretically and numerically (Chang et al. 2011 Meyrand \& Galtier 2010, Podesta et al. 2010, Camporeale \& Burgess |2011; Howes et al. 2011a; Sahraoui et al. 2012 Meyrand \& Galtier 2012). Determining the nature and properties (e.g., scaling, anisotropy) of the turbulence at small scales is indeed crucial to understanding the problems of energy dissipation and heating, particle acceleration, and magnetic reconnection in space and astrophysical plasmas (Schekochihin et al. 2009). Thanks to the high time resolution Search-Coil Magnetometer (SCM), which provides waveforms of the magnetic field fluctuations up to $180 \mathrm{~Hz}$, it has become clear that SW turbulence does cascade below the ion scale $\rho_{i}$ down to the electron scale $\rho_{e}$ where dissipation becomes important and the spectra steepen significantly. However, the underlying physics is still very controversial, owing to the fact that the available observations are very few and

fouad.sahraoui@lpp.polytechnique.fr the theoretical and numerical work has been extended to those small scales only very recently (Chang et al. 2011; Camporeale \& Burgess 2011; Howes et al. 2011a Sahraoui et al. 2012; Meyrand \& Galtier 2012).

From the observational point of view only a handful of observations exist on electron scales SW turbulence and all do not agree. The first point of controversy is the scaling of the magnetic energy spectra down to and below $\rho_{e}$. Sahraoui et al. (2009) have first reported a power-law cascade $f^{-2.8}$ down to $f_{\rho_{e}}$, where a clear spectral break is observed and followed by another power-law like shown to be close to $f_{s c}{ }^{-4}\left(f_{\rho_{e}}\right.$ is the frequency in the spacecraft frame corresponding to the electron gyroscale when the Taylor frozen-in-flow assumption is used ${ }^{1}$. It was emphasized in that reference that the fit below $f_{\rho_{e}}$ is subject to caution owing to the limited extension of the electron dissipation range, imposed by the sensitivity of the SCM (see section 2 below). Similar power-law-like spectra have been reported in Sahraoui et al. (2010a); Kiyani et al. (2009). On the other hand Alexandrova et al. $(\overline{2009})$, using STAFF-Spectrum Analyzer (STAFF-SA) data, have reported exponential scaling $\sim \exp \left(-\sqrt{k \rho_{e}}\right)$ in the scale range $k \rho_{e} \sim[0.1,1]$. More recently Alexandrova et al. (2012) proposed a different model where a scaling $k_{\perp}{ }^{-8 / 3} \exp \left(-k_{\perp} \rho_{e}\right)$ was suggested to fit better the data in the scale range $k \rho_{e} \sim[0.03,3]$. We will refer to the above three models respectively by the double-powerlaw, the exponential and the hybrid. Besides these three models we introduce below a new one, the asymptoticdouble-power-law. We show that the exponential model does not fit well the data as does the double-power-law model. We show however that, due to Cluster instru-

\footnotetext{
${ }^{1}$ It is commonly referred to as the Doppler-shifted electron gyroradius, although it is not a very appropriate definition considering that Doppler shift applies to frequencies and not to spatial scales. Here we introduce the terminology of the "Taylor-shifted scale".
} 
mental limitations that we shall discuss in detail, this latter may not be always possible to distinguish within the data from the two remaining models, the main difference between them being reduced to the presence or not of the spectral break near $\rho_{e}$.

From the theoretical point of view, very recent numerical simulations have tried to tackle the problem of cascade and dissipation at electrons scales. Camporeale \& Burgess (2011) performed 2D full Particle-In-Cell (PIC) simulations of decaying electromagnetic fluctuations near and below the electron inertial length $d_{e}$. They showed that the magnetic energy spectra steepen from $k_{\perp}^{-2.6}$ to $k_{\perp}^{-5.8}$ with a clear spectral break at $k \rho_{e} \sim 1$. 3D PIC simulations of whistler-driven turbulence showed very similar results with less steep spectra, $k_{\perp}{ }^{-4.3}$, at scales $k_{\perp} d_{e} \gtrsim 1$ (Chang et al. 2011). GyroKinetic (GK) simulations of strong Kinetic Alfvén Wave (KAW) turbulence, which self-consistently contain kinetic damping of the low frequency plasma modes $\left(\omega<<\omega_{c i}\right)$ but the cyclotron resonance, showed clear power-law cascade $k_{\perp}^{-2.8}$ down to $k_{\perp} \rho_{e} \sim 1$ (Howes et al. 2011a). The GK theory predicts magnetic energy spectra with a scaling $k_{\perp}^{-16 / 3}$ for the (entropy) cascade below $\rho_{e}$ (Schekochihin et al. 2009). On the other hand, Meyrand \& Galtier (2010) showed that incompressible Electron MHD (EMHD) turbulence predicts that the magnetic energy spectra should follow a power law $k^{-11 / 3}$ at scales smaller than $d_{e}$. That steepening was proposed to explain the observed spectra below the electron scale reported in the SW (Sahraoui et al. 2009, 2010a). Although this fluid model is nondissipative and does not consider any damping of the turbulence via kinetic effects, which are important in the dispersive and the dissipation ranges, it has nevertheless the merit of proving that fully nonlinear dynamics and dispersive effects in EMHD leads to a steepening of the energy spectra near $d_{e}$. Thus, all existing theoretical and numerical predictions support the doublepower-law model to reproduce the scaling near and below the electron gyroscale or inertial length. In a recent paper by Howes et al. (2011c) it was pointed out that for $\beta_{i}<<1$ the kinetic damping of the KAW turbulence becomes strong, which results in a cut-off of the spectra at moderate wavenumber, typically $k_{\perp} \rho_{i} \sim 10$. This is inconsistent with SW observations (Sahraoui et al. 2009 . 2010a Alexandrova et al. 2009). To overcome the failure of the model to reproduce SW observations it was suggested in that paper that nonlocal effects, due to large scale shear flows, play a role in sustaining the energy cascade down to smaller scales. When such nonlocal effects are considered it was indeed shown that the energy cascade in low $\beta_{i}$ can reach scales $k_{\perp} \rho_{e} \sim 1$, where the spectra curve and show an exponential-like behaviour. For $\beta_{i} \gtrsim 1$ relevant to the SW both the local and the nonlocal models give the same power-law scaling of the magnetic energy spectra down to $k_{\perp} \rho_{e} \sim 1$ (Howes et al. 2011a c), which may indicate that nonlocal effects do not play an important role in this case. We will return to this discussion below.

Here we report a large statistical survey of SW turbulence using the Cluster SCM data sampled at $450 \mathrm{~Hz}$. We focus particularly on the scaling of the magnetic energy spectra up to and above $f_{\rho_{e}}$, the frequency correspond- ing to Taylor-shifting the electron gyroscale $\rho_{e}$. In section 2 we discuss several caveats that need to be handled carefully in order to avoid any misinterpretation of the observations. In section 3 we explain the approach we used to select the Cluster data in the SW. We particularly target intervals when the Cluster fleet is located in the SW, excluding thus data from the electron and ion foreshock regions, and when the Signal-to-Noise-Ratio (SNR) of the magnetic fluctuations is high. In section 5 we show the main results of the study and discuss them in light of existing theoretical and numerical predictions and earlier work related to the subject.

\section{EXPERIMENTAL CAVEATS}

\subsection{Effect of the limited sensitivity of the SCM}

The Cluster SCM data are so far the only available data allowing one to probe into electron scales of SW turbulence with relatively high SNR. The SNR (in $\mathrm{dB}$ ) is defined as a function of the measured frequency onboard the spacecraft (Sahraoui et al. 2010c)

$$
S N R\left(f_{s c}\right)=10 \log _{10}\left[\frac{\delta B^{2}\left(f_{s c}\right)}{\delta B_{\text {sens. }}{ }^{2}\left(f_{s c}\right)}\right]
$$

where $\delta B$ and $\delta B_{\text {sens. }}$ are respectively the amplitude of the magnetic fluctuation and the level of the sensitivity floor of the Cluster SCM at the frequency $f_{s c}$. Current space missions (e.g. Stereo, Wind, Themis) and the planned ones (e.g. MMS) either do not have SCM or do have but with limited sensitivity. The sensitivity of the SCM is essentially (but not exclusively) limited by the length of the magnetic sensor, which is generally constrained by the payload on the spacecraft. For instance the SCMs onboard Cluster, Themis and MMS have sensors with respective lengths of $27 \mathrm{~cm}, 15 \mathrm{~cm}$ and $10 \mathrm{~cm}$ which yields respective sensitivity levels of $0.43,0.70$, and $2.35 p T / \sqrt{H z} @ 10 H z$. Despite this relatively high sensitivity of the Cluster SCM compared to all other space missions, it does not allow us all the time to measure the SW magnetic fluctuations above $\sim 30 \mathrm{~Hz}$ due to their very low amplitude (see Figs. 7 and 8 in Sahraoui et al. (2010c)). Indeed, when the measured SNR is exceptionally high (typically SNR $\gtrsim 30$ ) one should suspect the crossings of other regions such as the ion or electron foreshock (Sahraoui et al. 2009) or boundaries such as interplanetary shocks or CMEs (e.g. the event of 2004-01-22 studied in Alexandrova et al. (2009, 2012)). We recall that Cluster, Themis and MMS are magnetospheric missions and have been designed to address essentially the physics of different regions of the Earth's magnetosphere and the magnetosheath where the magnetic fluctuations have generally higher amplitudes than in the SW.

It follows from this discussion that in order to address properly the problem of energy cascade and dissipation in the SW at electron scales, typically at frequencies $f_{s c} \gtrsim 30 \mathrm{~Hz}$, it is important to select SW data when the fluctuations amplitude is very high compared to the sensitivity floor of the Cluster SCM. Because of this limited SNR in the SW Alexandrova et al. (2009) proposed to subtract the sensitivity level of the instrument from the measured power spectra. Without discussing in detail the foundation of a such procedure from the signal processing point of view (in particular since the 
noise of the instrument is not known accurately, and is generally estimated from measurements in the magnetospheric lobes), we only underline how affected would be the scaling of the resulting "denoised" spectra. Indeed, when the SNR becomes very low at a given frequency $f_{0}$, i.e. $\delta B^{2}\left(f \gtrsim f_{0}\right)=(1+\epsilon(f)) \delta B_{\text {sens }}^{2}\left(f \gtrsim f_{0}\right)$ with $\epsilon(f)<<1$, subtracting the sensitivity of the instrument from the actual spectrum yields a spectrum which falls to zero: $\delta B^{2}-\delta B_{\text {sens }}^{2} \sim \epsilon \delta B_{\text {sens }}^{2}$. It is not surprising that on the Log-Log scale such a vanishing spectrum will be seen as exponentially falling-off for all frequencies $f \gtrsim f_{0}$.

\subsection{Effect of the limited frequency resolution of STAFF-Spectrum Analyzer}

The STAFF instrument onboard Cluster has a single tri-axial Search-Coil Magnetometer (SCM), but has two sub-experiments: STAFF-SC measures magnetic field fluctuations (i.e. waveforms) with two possible sampling time: 25 samples/sec (Normal Mode, NM) and 450 samples/sec (Burst Mode, BM) with however a low-pass filter cut-off at $180 \mathrm{~Hz}$, and STAFF-SA computes onboard the spacecraft the $5 \times 5$ spectral matrix of the three components of the magnetic field and the two components of the electric field fluctuations measured by EFW (Cornilleau et al. 2003). STAFF-SA transmits the computed correlation matrix on the central frequencies $f_{0}$ of 27 channels logarithmically spaced in the frequency range $[8 \mathrm{~Hz}, 4 \mathrm{kHz}]$ whose bandwidth is proportional to $f_{0}, \Delta f=13 \% f_{0}$. In $\mathrm{BM}$ the interval reduces to approximately $[70 \mathrm{~Hz}, 4 \mathrm{kHz}]$. It is important to emphasize here that due to the limited SNR in the SW, which generally does not allow us to address frequencies (in the spacecraft frame) higher than $\sim 100 \mathrm{~Hz}$ and considering that the Taylor-shifted electron gyroscale is typically $f_{\rho_{e}} \sim 70 \mathrm{H}^{2}$, STAFF-SA provides thus information only at a few frequencies in the interval $[8,100] \mathrm{Hz}$ which are $\sim[9,11,14,18,22,28,35,44,55,70,88] \mathrm{Hz}$. This limited frequency resolution of the STAFF-SA spectra in the scale range between proton and electron gyroscales may result either in missing the spectral breaks or in not localizing them properly. In both cases determination of the scaling of the spectra at electron scales may be not very accurate. To show such a difficulty to locate the spectral break we plotted in Fig.1 the same spectrum as in Fig 8(c) (measured by STAFF-SC) as it would have been measured by STAFF-SA. We can see that while the spectral break can be observed easily on Fig 8 (c) (at $f \sim 30 \mathrm{~Hz})$ it becomes more difficult to identify in Fig. 1 . The limited number of available points $(\sim 10)$ would also make any fit subjet to high uncertainties (i.e. large error bars).

This effect may be particularly relevant to explain the very low correlation $(C=0.03)$ between the spectral breaks and $\rho_{e}$ reported in (Alexandrova et al. 2012) as compared to the one shown in Fig $10(C=0.72)$, which is obtained using the STAFF-SC BM waveforms. STAFF$\mathrm{SA}$ is certainly useful to provide information on the overall shape of the spectra at high frequency. However, it

${ }^{2}$ For SW speed of $\sim 600 \mathrm{~km} / \mathrm{s}$. Faster SW yields higher values of $f_{\rho_{e}}$ that can be larger than $100 \mathrm{~Hz}$. In this case using STAFFSA spectra may prove to be useful to study the electron dissipation range forming above $f_{\rho_{e}}$.

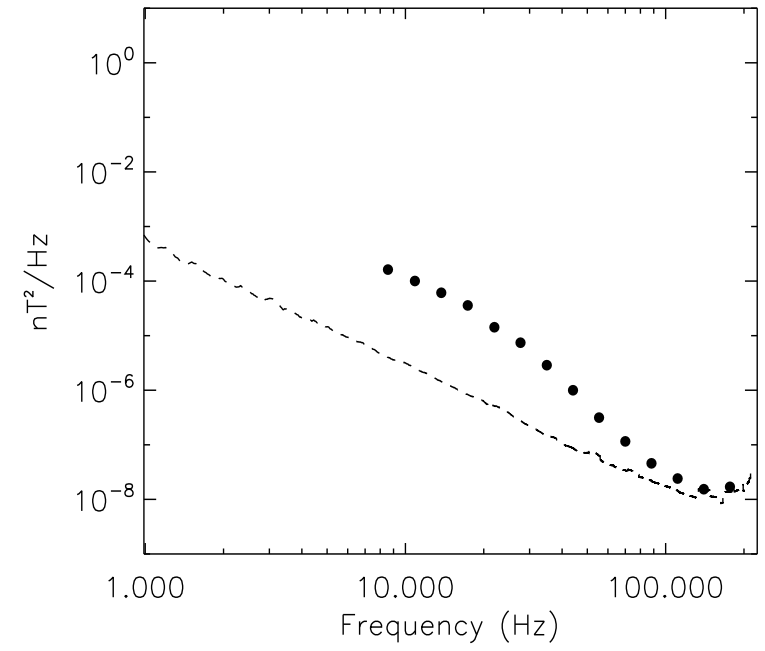

FIG. 1. - The spectrum of Fig 8 (c) as it would have been measured by STAFF-SA with the avallable sampling frequencies.

may not suffice to address specific details such as the actual scaling of the spectra at electron scales. When available, BM STAFF-SC data is certainly more appropriate to address this problem. The available range of frequencies $([1,180] \mathrm{Hz})$ is generally sufficient to address electron scale physics. In cases when electron scales would correspond to higher frequencies in the spacecraft frame (e.g. fast SW, or quasi-parallel whistler-like fluctuations) not accessible to STAFF-SC one then may use STAFF-SA spectra to probe into electron scales (provided that the SNR remains sufficiently high at those frequencies).

\subsection{Effect of averaging the power spectra}

Another effect that may also influence the determination of the scaling of the turbulent spectra at electron scales is the time (or frequency) averaging of the spectra, which is usually done to smooth the spectra under the assumption of time stationarity of the fluctuations. The approach consists generally in dividing a given time series into several shorter intervals of time for which a power spectrum is computed. Then the resulting spectra are averaged to reduce the fluctuations noise in particular at high frequencies. However, considering that the turbulent fluctuations are not stationary strictly speaking, averaging the spectra may result in missing some of the information contained in the individual spectra. This is particularly true regarding the presence of a spectral break at electron scales. For instance, let us assume that each given subinterval of time yields a power spectrum with a clear breakpoint at a given frequency $f_{b}$ in the spacecraft reference frame. If the value of the frequency $f_{b}$ changes from one subinterval of time to the other, due for instance to fluctuations either in the mean SW parameters (e.g. $\left.V_{s w}, T_{i}, T_{e}, V_{A}\right)$ that enter in estimating the scale $\rho_{e}$ or the frequency $f_{\rho_{e}}$ corresponding to it and if the individual spectra have comparable power, then averaging them will result in smoothing the spectral breaks yielding a curved-like spectrum in the frequency range corresponding to all the breaks. To show this we plotted in Fig. 2 (top panel) three spectra computed over $10 \mathrm{sec}$ within a window of $10 \mathrm{mn}$. The three spectra show clear breakpoints approximately at $20 \mathrm{~Hz}, 30 \mathrm{~Hz}$ and $45 \mathrm{~Hz}$. When the three spectra were averaged (bottom panel) they yield a spectrum without any clear break 

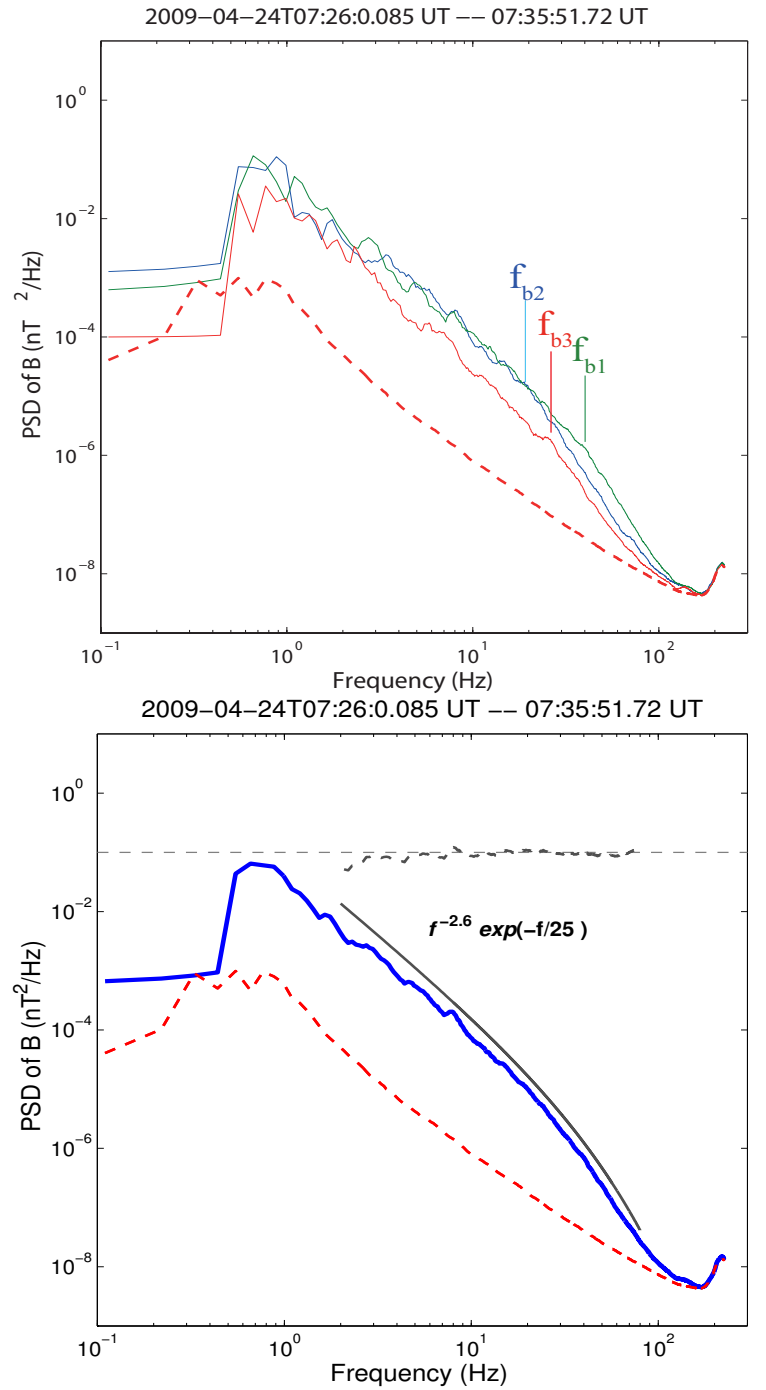

FIG. 2.- Three magnetic energy spectra measured in the SW showing breakpoints at different frequencies indicated by the vertical lines (top). The mean spectrum shows no clear break and curves at those frequencies (bottom). The horizontal curve (bottom panel) shows the compensated spectrum $B^{2}(f) / P(f)$ where $P(f)$ is the used fitting function.

in the frequency range $[20,45] \mathrm{Hz}$. The spectrum at those frequencies curves and can be well fit by exponential-like models.

\subsection{Effect of instrumental interferences at high frequency}

The last instrumental problem that can affect the proper determination of the scaling the magnetic energy spectra in the SW is the presence of spectral interferences observed at high frequency of STAFF-SC spectra. These interferences may be caused by the Digital Wave Processor (DWP) onboard Cluster. The DWP instrument is designed to synchronize the wave instruments of Cluster by emitting a signal allowing communication between them. This signal might be at the origin of the interferences with the STAFF magnetometers (similar interferences are also observed on FGM spectra). Examples of such interferences are shown in Fig. 3.

Although the spikes caused by such interferences are few, their relatively high amplitudes may nevertheless
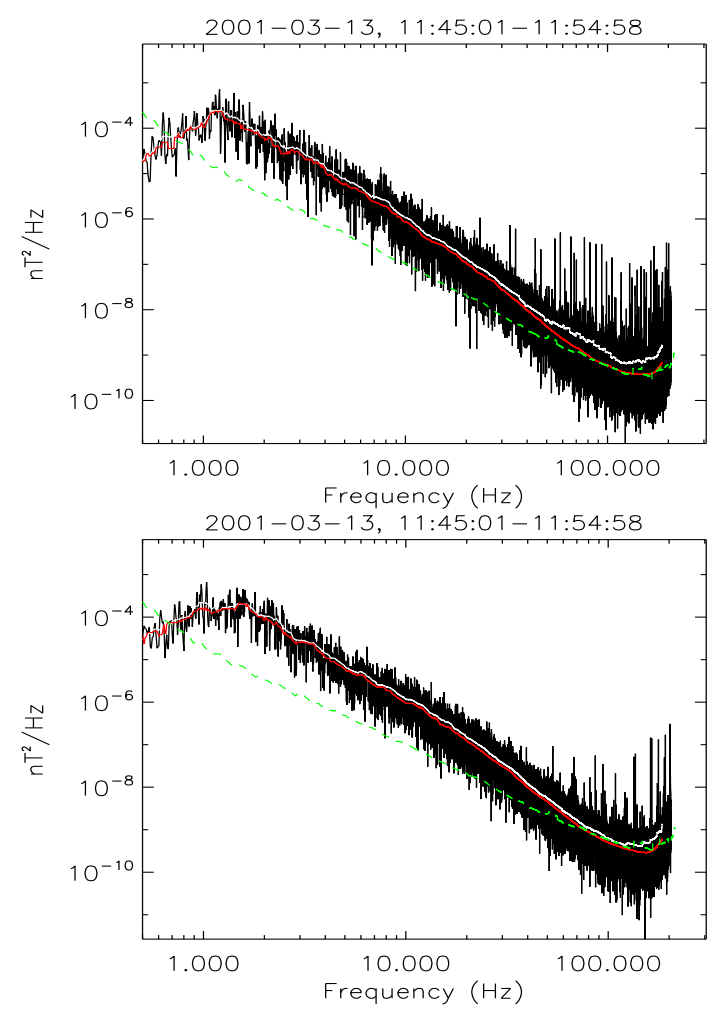

FIG. 3.- An example of high frequency spikes observed on the spectra of $B_{z}$ measured by the STAFF instrument which may be caused by interferences coming from the DWP instrument onboard spacecraft 1 (top) and spacecraft 2 (bottom). The corresponding smoothed spectra by a mean (white) and the median (red) functions are shown. The dashed green line is the in-flight mean sensitivity floor of the STAFF instrument.

affect the fit of the turbulent spectra at electron scales. Indeed, when smoothed the intense spikes lead to over estimating the power at the highest part of the spectra, which then produces shallower spectra as shown in Fig. 3 (top). The figure compares smoothings by a mean and a median functions on windows of size logarithmically increasing with frequency. The effect of interferences becomes more important for spectra with low SNR, which emphasizes the need to use the highest available SNR in the SW.

From Fig 3 we can also see that the four Cluster satellites are not affected equally by the interferences. A more complete study (not shown here) revealed that spacecraft 1 , and particularly its $B_{z}$ component, is most affected. Therefore, considering that most of the studies of the electron scales in the SW are based on single spacecraft data we recommend to use data from the spacecraft 2,3 or 4. Depending on the level of the SNR, the number and amplitudes of the spikes that would appear on the spectra, the corresponding data may need to be pre-processed by removing the spikes prior to any analysis.

Finally, we note that these interferences would also affect the spectra computed by the STAFF-SA instrument onboard the spacecraft. Owing to the fact that the STAFF-SA spectra are averaged over a frequency band centered at each of the 27 frequencies, the contribution of the spikes may be accounted for in the transmitted energy density. Since the original waveforms are lost, it is not possible to quantify the effect of the interferences on the STAFF-SA spectra. 


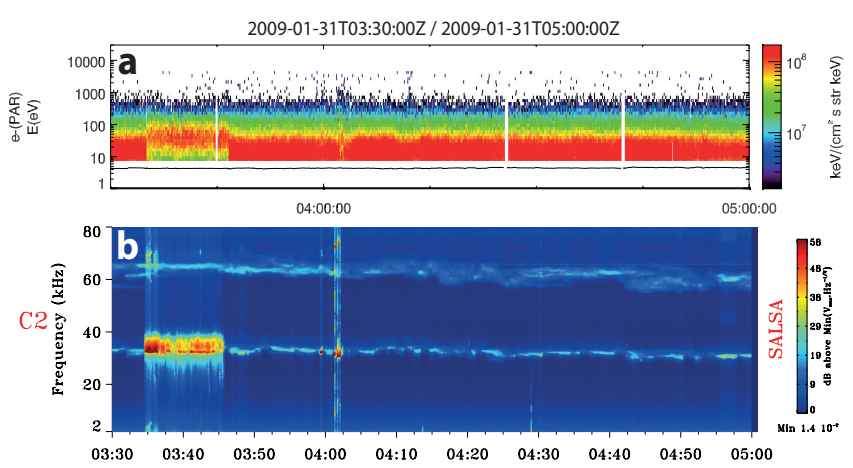

FIG. 4. - A typical example of the analyzed events. (a) is the spectrogram of field-aligned electron energy and (b) is the electric field from PEACE (SC1, no data available on SC2) and WHISPER (SC2) showing that the spacecraft are in the free SW from 04 h05 to $05 \mathrm{~h} 00$ (no foreshock electrons).

\section{DATA SELECTION}

In the present study we surveyed all STAFF-SC BM data measured in the SW by the Cluster 3 spacecraft from 2000 to 2011. We used AMDA data base (http://cdpp-amda.cesr.fr/DDHTML/index.html) to select intervals of time when the Cluster spacecraft was located in the SW. AMDA provides useful routines to search for data according to desired physical parameters (e.g., high or low SW speeds, plasma $\beta$ ). The obtained list of events is then checked event by event with respect to WHISPER and PEACE data to remove any interval containing foreshock electrons. An example of the targeted SW intervals is shown in Fig 4 .

To eliminate data from the ion foreshock region (IFS), or from other boundaries such as Interplanetary Shocks (IPS) or CMEs requires additionally to use data from the Cluster Ion Spectrometer (CIS). The free SW is indeed generally characterized by a beam of ions with an energy around $1-2 \mathrm{KeV}$. Any enlargement of the beam toward higher energies generally indicates a heating of ions across boundaries such as shocks. The CIS spectrograms available at the ESA Cluster Active Archive (CAA) web site (http://caa.estec.esa.int/caa/home.xml) allow one to identify the IFSs and IPSs. However, the spectrograms cannot guarantee the absence of any reflected ions from the shock, which can only evidenced by a careful analysis of the Ion Distributions Functions (IDFs). In the following, to eliminate the significant EFSs, IFSs and IPSs we rely mostly on the examination of the Whisper, PEACE and CIS spectrograms. If afterwards a doubt still remains we then analyze the corresponding IDFs provided at the CAA web site to check the presence of reflected energetic ions from the foreshock ${ }^{3}$ Despite these numerous checks of the data one still needs to assume that if any residual reflected ions from the foreshock would still exist in our data (not observable on the spectrograms or in the analyzed IDFs) they do not have significant impact on the electron scale physics, which is the main purpose of the present paper. Indeed, such ions when they exist would affect ion scales $\sim \rho_{i}$ (rather than electron scales $\sim \rho_{e}$ ) through, for instance, ion plasma instabilities that might be generated

3 The IDFs given in the ESA/CAA website may need a careful post-treatment to obtain clean IDFs in the SW. However, doing such detailed studies of the IDFs is hardly feasible for all our events (Mazelle 2012).

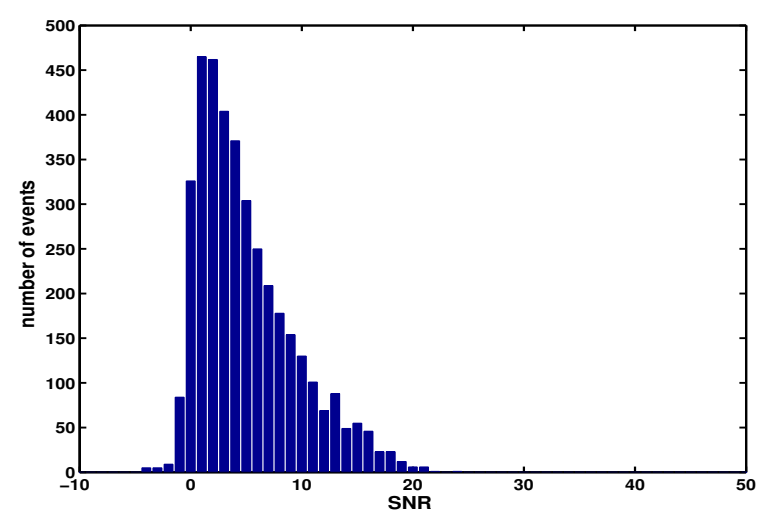

FIG. 5.- The distribution of the Signal-to-Noise-Ratio (defined in the text) of the $B_{z}$ magnetic energy spectra at $f=30 \mathrm{~Hz}$.

in the SW plasma.

After obtaining the list of events that survive the different criteria given above, we focus only on intervals where magnetic fluctuations have a high SNR. To do that we computed the magnetic energy spectra over approximately 10 s for all intervals and estimated the value of the SNR at the frequency $f_{s c} \simeq 30 \mathrm{~Hz}$. With a $\mathrm{SW}$ speed $V_{s w} \sim 500 \mathrm{~km} / \mathrm{s}$ and an electron gyroradius $\rho_{e} \sim 1 \mathrm{~km}$, the frequency $f_{s c}=30 \mathrm{~Hz}$ corresponds roughly to the scale $k \rho_{e} \sim 0.4$ using the Taylor assumption (other histograms of SNR were computed at neighboring frequencies and found to be very similar to the one shown here). The obtained histogram (Fig. 5) shows that at $f_{s c} \simeq 30 \mathrm{~Hz}$ most of the turbulent spectra reach the sensitivity floor of the Cluster SCM, which means that those events cannot be used to address the electron scale physics. To achieve this goal we need to select intervals with the highest possible SNR. However, as can be seen on Fig. 5, the higher is the SNR the rare are the events. Here we present only the results for events having $\mathrm{SNR} \geq 10$, and a number of 620 was found. These events represented about $15 \%$ of the total events (an "event" refers to a total power spectrum computed over nearly 10 s period of time).

\section{FITTING MODELS}

As we discussed above, the theoretical and numerical studies of SW turbulence dissipation at electron scale are very recent. The absence of firm theoretical predictions on this problem and the limitations of the available data make it difficult to draw firm conclusions on the actual processes of dissipation, or even on the actual scaling of the energy spectra at electron scales. To illustrate that we plotted in Fig 6 an example of a magnetic energy spectrum measured in the SW by the STAFF-SC experiment (black curve). We also over plotted different models to fit the data:

- Double-power-law model

$P(f)=A_{1} f^{-\alpha_{1}}\left[1-H\left(f-f_{b}\right)\right]+A_{2} f^{-\alpha_{2}} H\left(f-f_{b}\right)$

- Exponential model

$$
P(f)=\operatorname{Aexp}\left(-a \sqrt{f / f_{b}}\right)
$$

- Hybrid model

$$
P(f)=A f^{-\alpha_{1}} \exp \left(-f / f_{b}\right)
$$




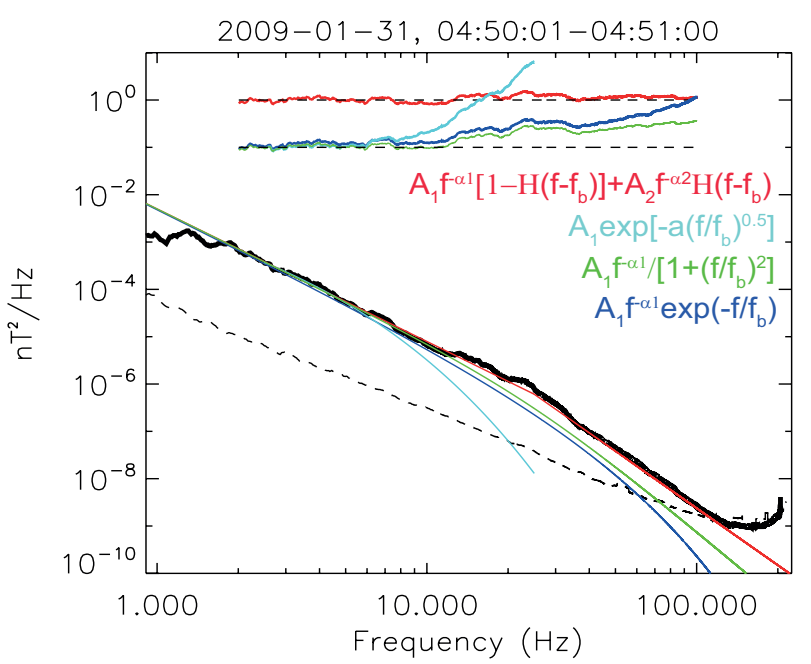

FIG. 6.- Example of a magnetic energy spectrum of $B$ measured in the SW by the Cluster 2/STAFF-SC instrument (black). The dashed line indicates the estimated (in-flight) sensitivity floor of the instrument. Examples of fitting functions are plotted for comparison: double-power-law (red), asymptotic-double-powerlaw (green), hybrid (blue) and exponential (cyan). The horizontal curves are compensated spectra $B^{2}(f) / P(f)$, where $P(f)$ is the corresponding fitting function.

- Asymptotic-double-power-law model

$$
P(f)=A \frac{f^{-\alpha_{1}}}{1+\left(f / f_{b}\right)^{2}}
$$

Above $H(f)$ is the Heaviside function, $f_{b} \sim 25 \mathrm{~Hz}$, $\alpha_{1}=2.6$ and $\alpha_{2}=4.2$, and $a=15$. The exponential model has been proposed in Alexandrova et al. (2009) to fit the magnetic energy spectra in the scale range $\sim[0.1,1] k \rho_{e}^{4}$ The hybrid model is reported in Alexandrova et al. (2012) and combines the two previous ones where the power-law dominates the lowest part of the spectrum while the exponential function starts acting as frequencies approach $f_{b}$ yielding a curved-like spectrum. The last model is asymptotically similar to the doublepower-law model: for $f<<f_{b} P(f) \sim A f^{-\alpha_{1}}$ and for $f>>f_{b} P(f) \sim f^{-\alpha_{1}-2} \sim f^{-4.6}$. However, when $f \sim f_{b}$ this model is very close to the hybrid model and results in a curved-like spectrum.

One should stress here that these functions are chosen for pure mathematical reasons and they do not necessarily reflect actual physical mechanisms of dissipation, although there may exist a possible connection between a given scaling with a particular dissipation process. For instance hydrodynamic turbulence is known to yield an exponential dissipation, while asymptotic-double-powerlaw models have been reported in fusion plasma turbulence where the Finite Larmor Effects (FLRs) play a key role (Hasegawa et al. 1978, Gurcan et al. 2009).

The comparison between the fits in Fig. 6 shows indeed that the double-power-law model fits better the data as it captures the observed spectral break. The exponential model is shown not to reproduce correctly the scaling in the range $k \rho_{e} \sim[0.1,1]$ as reported in Alexandrova et al. (2009). This model will not be considered in the rest of the paper. However, as expected, the hybrid and

4 To change from frequency to wavenumber one can replace $f / f_{b}$ by $k \rho_{e}$ using the Taylor hypothesis and assuming $f_{\rho_{e}} \sim f_{b}$.
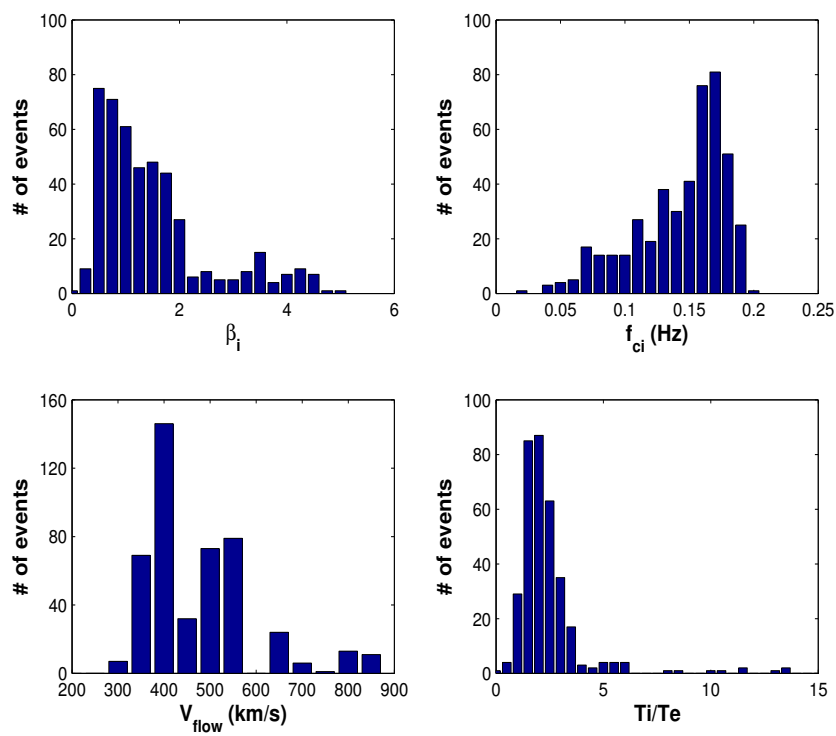

FIG. 7.- The histograms of different mean SW parameters: $\beta_{i}$, ion cyclotron frequency $f_{c i}$, the solar wind speed $V_{\text {flow }}$ and the ratio of ion to electron temperatures.

the asymptotic-double-power-law fail to fit the spectral break and the neighboring frequencies but they reproduce the overall shape of the spectrum, in particular the asymptotic-double-power-law. Nevertheless, these two models are very close to each other, significant differences appear indeed only at high frequency where the noise floor of the instrument is reached. From this one concludes that, given that the highest SNR values available from the Cluster/SCM data in the SW are at best similar to that of Fig. 6, it is unrealistic to hope distinguishing between these two models in SW observations. However, the double-power-law model can be distinguished from the three other (curved) models provided that the spectral break can be evidenced properly. As discussed above to detect the spectral break at electron scales requires particular caution as its presence may easily be smoothed by several effects.

\section{RESULTS}

For the obtained list of events we computed the histrograms of the corresponding mean plasmas parameters, which are given in Fig. 7. We found in particular that most of the events have $T_{i}>T_{e}$ and $\beta_{i} \gtrsim 1$. For these events we computed the corresponding power spectra of the magnetic fluctuations over $10 \mathrm{~s}$. This time length has been chosen to allow for covering the frequency range $\sim[1,180] \mathrm{Hz}$. To study lower frequencies longer time intervals are required, but these frequencies will not be addressed here.

Some examples of the obtained spectra are plotted on Fig. 8. They show that the magnetic energy cascades below the ion scale and reaches the electron gyroscale where a clear break is evidenced, in agreement with earlier observations reported in Sahraoui et al. (2009, 2010a). Similarly to Fig. 6, we plotted on Fig, 8 (a) the three previous fitting modes to check which one fits better the spectra. As can be seen on the compensated spectra plotted on the top of the figure, the double-power-law is found to fit better the observed spectra at the scales near and below the spectral break. The presence of a clear break 

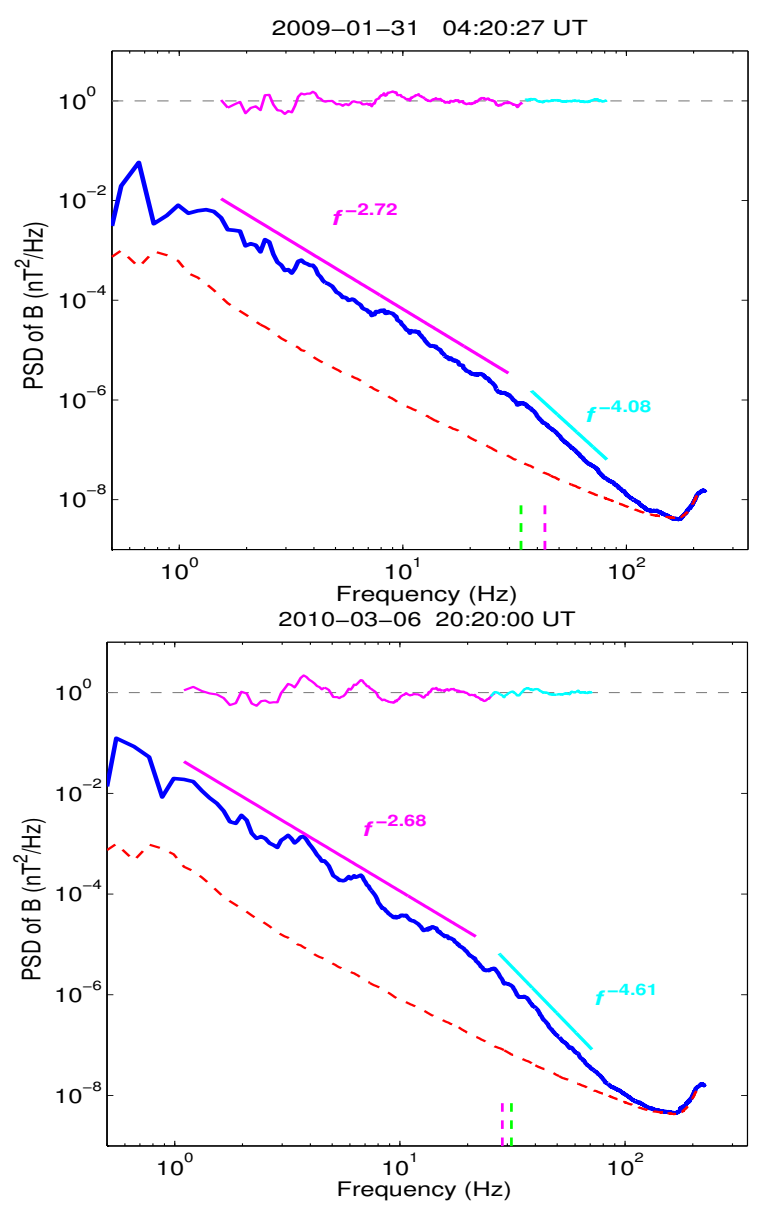

FIG. 8. - Examples of the magnetic energy spectra measured in the free SW showing clear spectral break at electron scales (vertical dashed lines). A similar description as in Fig 6 applies.

near the electron scales invalidates the argument that the break at electron scales is essentially caused by the foreshock electrons present in the data analyzed in Sahraoui et al. (2009). Indeed, the presence of foreshock electrons may generate a "bump" in the magnetic spectra at that scale as shown in Fig.3(a) in Sahraoui et al. (2009), but it is not clear as to how they can generate a spectral break followed by a steep power-law spectrum as shown in Fig.3(b) of Sahraoui et al. (2009). In any case, the results shown here, which were obtained from SW data that contained no foreshock electrons clearly prove that the spectral break cannot be attributed primarily to foreshock electron. It most likely results from the nonlinear dynamics of the plasma itself. This indeed has been observed in 2D and 3D PIC simulations (Camporeale \& Burgess 2011, Chang et al. 2011), predicted by existing theories on SW turbulence (Schekochihin et al. 2009. Meyrand \& Galtier 2010) and observed also in magnetosheath turbulence (Huang et al. 2013). The disagreement with the observations of Alexandrova et al. (2009) can be explained by the lack of universality of turbulence at electron scales, or by the caveats discussed in section 2 , Note however that the recent study by Alexandrova et al. (2012) showed also that $30 \%$ (out of 100 ) of the analyzed STAFF-SA spectra agreed with the double-power-law (or the break) model discussed here.

Following the arguments given here we performed similar double-power-law fits below and above the spectral
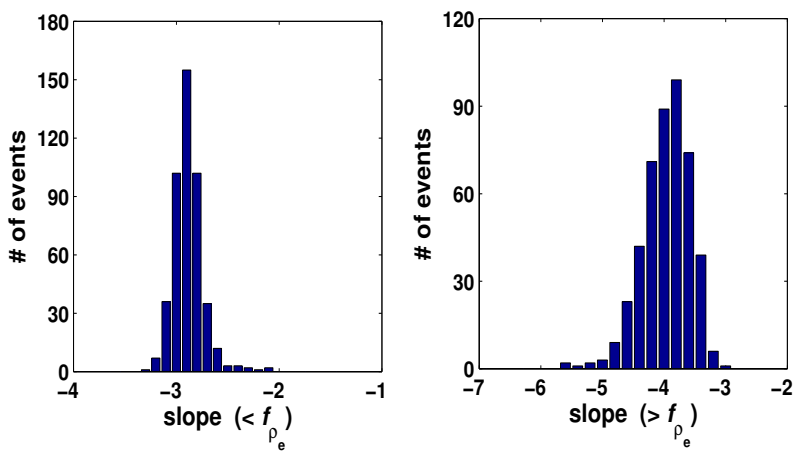

FIG. 9. - The histograms of the slopes resulting from a doublepower-law fits of the scales below (left) and above (right) the frequency $f_{b}$ corresponding to the spectral break near the electron gyroscale.

break to all events found to have breaks (some events, mostly those corresponding to fast SW, do not show breaks in the frequency range accessible to STAFF-SC). The histograms of the obtained slopes are given in Fig. 9 We can see that the slopes of the spectra in the dispersive range (i.e. $\left[f_{\rho_{i}}, f_{\rho_{e}}\right]$ ) cover the range $\sim[-2.5,-3.1]$ with a peak at $\sim-2.8$. These values are close to those predicted by existing theoretical and numerical simulations (Camporeale \& Burgess 2011; Chang et al. 2011; Howes et al. 2011a Boldyrev \& Perez 2012) and to the observations reported in Alexandrova et al. (2012). The spectra below $f_{\rho_{e}}$ are however steeper with slopes distributed in the range $\sim[-3.5,-5.5]$ and a peak at $\sim-4$, which are in general agreement with the predictions from the 2D and the 3D PIC simulations (Camporeale \& Burgess 2011; Chang et al. 2011) and with the results of the break model reported in Alexandrova et al. (2012). This wide distribution suggests the lack of universality of turbulence at electron scales as compared to that in the inertial range. A similar study has been performed on magnetosheath turbulence where steeper spectra above $f_{\rho_{e}}$ were observed whose slopes distributed in the interval $\sim[-4,-7.5]$ and a peak near -5.5 (Huang et al. 2013). We will discuss this point further below.

Another important question that we can try to answer here is the actual physical scale, $\rho_{e}$ or $d_{e}$, that would correspond to the spectral breaks observed within the data. This question relates to the appropriate theoretical description of the electron scales physics (Schekochihin et al. 2009, Bourouaine et al. 2012). In the so-called Hall-MHD approximation, which is valid in the limit of $\beta_{i}<<1$ and $T_{i}<<T_{e}$, the Hall term brings a correction to the classical MHD theory proportional to $d_{i}$, the ion inertial length. This theory can be extended to electron scale physics within the incompressible Electron-MHD (EMHD) (Biskamp et al. 1999; Meyrand \& Galtier 2010) or the reduced two-Hluid-theory (Sahraoui et al. 2003b 2012). Within the EMHD model, which is appropriate to describe whistler mode turbulence, $d_{e}$ arises as the relevant scale to describe electron scales physics. However, in the SW at $1 \mathrm{AU}$, where $\beta_{i} \sim 1$ and $T_{i} / T_{e} \gtrsim 1$ (see Fig, 7), these popular models are not rigorously valid (Schekochihin et al. 2009, Howes 2009), although they may reproduce some observed properties of SW turbulence such as the scaling of the magnetic or the electric energy spectra in the dispersive range (Matthaeus et al. 2008). 

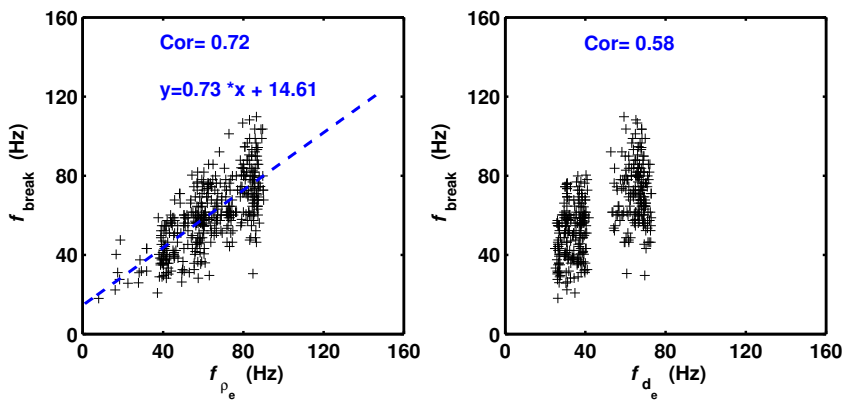

FIG. 10.- Correlation between the frequencies of the observed spectral breaks $f_{b}$ with the Taylor-shifted electron gyroscale $\rho_{e}$ (right) and inertial length $d_{e}$ (left).

In kinetic theory, 3D recent PIC simulations of electron scales whistler turbulence showed that a spectral break occurs near $d_{e}$ (Chang et al. 2011) and not at $\rho_{e}$ (note that in that paper $\beta_{e}=0.1$, which means that $\rho_{e}=$ $\left.d_{e} / \sqrt{\beta_{e}} \sim 3 d_{e}\right)$. On the other hand kinetic theory of KAW turbulence in the limit $k_{\|}<<k_{\perp}$ predicts that the relevant scales are $\rho_{i}$ and $\rho_{e}$. A simple explanation of this can be given by examination of the linear dispersion relations of the KAW at different values of $\beta_{i}$ (or $\beta_{e}$ ) shown in Fig. 13 (top). One can see that regardless the value of $\beta_{i}$ the KAW becomes dispersive at $k \rho_{i} \sim 1$ and no significant change is observed at $k d_{i} \sim 1$.

From the point of view of observations answering the question as to whether $\rho_{i}$ or $d_{i}$ is the relevant scale is more difficult because, often, SW data at $1 \mathrm{AU}$ show that $\beta_{i} \sim 1$, which means that one cannot distinguish the two scales. Nevertheless, some statistical studies have tried to answer this question (Leamon et al. 1999; Bourouaine et al. 2012). For electron scales only a few studies based on a limited number of events have reported the relevance of $\rho_{e}$ to characterize electron scale physics in the SW (Sahraoui et al. 2009, Alexandrova et al. 2009, Sahraoui et al. 2010a). Alexandrova et al. (2012) have confirmed that result over a larger data samples (100 spectra). In Fig. 10 we computed the correlation between the frequencies of the observed spectral breaks with $f_{\rho_{e}}$ and $f_{d_{e}}$. The figure shows a relatively high correlation $(0.72)$ of the spectral break with the electron gyroscale $\rho_{e}$ than with the inertial length $d_{e}$. This result contrasts with the result in Alexandrova et al. (2012) which showed no correlation $(C \sim 0.03)$ between the spectral breaks (given by the used double-power-law model) and $\rho_{e}$. A possible explanation of the lack of the correlation in that work is the downsampling frequency of the STAFF-SA that does not allow one to capture properly the spectral breaks. We note that the moderate correlation with $d_{e}(0.58)$ maybe due to all intervals that have $\beta_{e} \sim 1$ (for those events $\left.\rho_{e} \sim d_{e}\right)$. We note finally that a similar correlation coefficient between $f_{b}$ and $\rho_{e}$ is found in magnetosheath turbulence (Huang et al. 2013), but a weaker correlation is found with $d_{e}(C=0.27)$. All these observations suggest the relevance of $\rho_{e}$ as a dissipation scale for the SW and in the magnetosheath.

\section{DISCUSSION}

The results shown in the previous section underline the need to carefully handle the Cluster/STAFF data in the SW in order to analyze properly the energy spectra at electron scales. We showed in particular that in order to evidence the presence of the spectral breaks at electron scales time series with high SNR are needed. For the selected data a large number of magnetic energy spectra was found to have a clear break near the electron scale, followed by steep power-law-like spectra $f^{\alpha}$. The distribution of their slopes $\alpha$ was shown to range from -3.5 to -5.5 with a peak near -4 . This distribution is wider than that of the slopes in the dispersive range which covers the values $-3.2 \lesssim \alpha \lesssim-2.3$. A comparison of these distributions to those reported in magnetosheath turbulence (Huang et al. 2013) shows some interesting similarities and differences. In the dispersive range the distribution of the slopes is very similar: both are narrow and show a peak near -2.8 , in general agreement with most of theoretical and numerical predictions on SW turbulence. However, in the electron dissipation range the magnetosheath spectra were found to be generally steeper with slopes $\alpha \sim-7$, whose distribution peaks near -5.5 . This comparison stimulated a further analysis of the data to explain the difference between the SW and magnetosheath observations based on the differences in the SNRs between the two regions. Let us examine this effect in detail.

Although the data used here have the highest found SNR in ten years of data survey in the SW, it might be possible that even SNR values as high as 10 are not sufficient enough to determine accurately the scaling above $f_{b}$. The reason is the limited extension of the spectrum above $f_{b}$ (typically less than one decade) due to the proximity of the sensitivity floor of the SCM. To test this hypothesis we plotted in Fig. 11 the measured slopes of the spectra above $f_{\rho_{e}}$ as a function of the corresponding SNR. The figure shows a moderate correlation $(C=0.53)$ between the SNR and the slopes. Typically most of the slopes $|\alpha| \lesssim 3.5$, which were not observed in magnetosheath turbulence, correspond to $\mathrm{SNR}<15$ and the highest values of the slopes $|\alpha| \gtrsim 4.5$ correspond to $\mathrm{SNR} \gtrsim 15$. This correlation suggests that data with even higher SNR are needed to fully address the actual scaling of the magnetic energy spectra at sub-electron scales in the SW. Based on this conclusion the powerlaw fits and the interpretation of the low values of slopes $(|\alpha| \lesssim 3.5)$ reported in this study should be considered with some caution. We emphasize that a weak correlation $(C<0.1)$ is found in magnetosheath turbulence when SNR $>25$ (Huang et al. 2013).

Another interesting point that is worth a discussion is the possible dependence of the scaling of the magnetic energy spectra in the dispersive and the dissipation ranges on the plasma $\beta_{i}$. If one assumes that turbulence below the ion scale is dominated essentially by high oblique KAWs as suggested by several SW observations (Bale et al. 2005; Sahraoui et al. 2009, 2010a; Salem et al. 2012, Kiyani et al. 2013) such a dependence could exist. This is indeed based on the known result from the linear kinetic theory that the damping of high oblique KAWs depends strongly on the plasma $\beta_{i}$. This can be seen clearly in Fig. 13 (top panel) which shows that the lower is the $\beta_{i}$ the more damped is the KAW mode (for the same angle of propagation). Based on this remark one would expect to observe a rapid decay of the energy spectra at low $\beta_{i}$, which would translate into steeper power-law or exponentially decaying spectra (Howes et al. 2011c). To test this hypothesis we plotted on Fig. 12 the correlation between the observed slopes below and above 


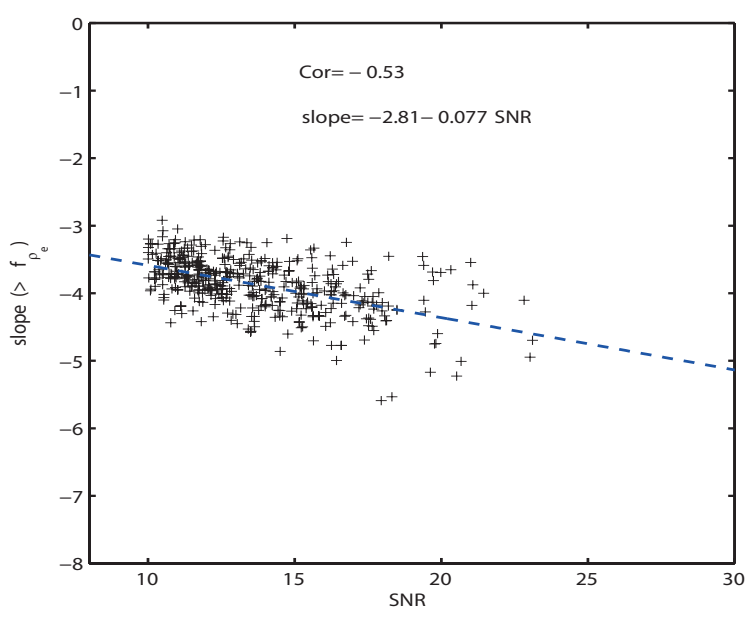

FIG. 11.- Correlation between the frequencies of the observed spectral breaks $f_{b}$ with the SNR (see text) at $30 \mathrm{~Hz}$ of the studied magnetic energy spectra.
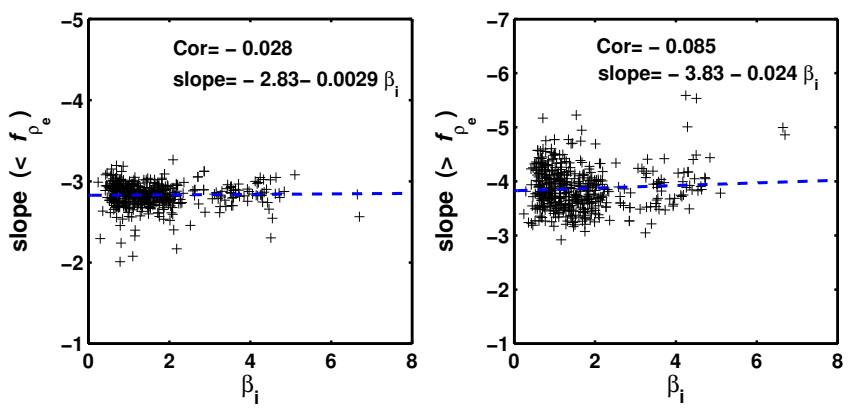

FIG. 12.- Correlation between the frequencies of the observed spectral breaks $f_{b}$ with the plasma $\beta_{i}$ of all analyzed time intervals.

$f_{\rho_{e}}$ as a function of $\beta_{i}$. The results do not show any clear dependence between the values of the slopes and $\beta_{i}$. A possible explanation of that is the fact that the difference in the kinetic damping of the KAW modes is more pronounced between $\beta_{i} \sim 1$ and much lower values $\beta_{i} \sim 0.01$. This cannot be seen directly on Fig. 13 (top). To evidence this fact plotted in Fig. 13 (bottom) the damping of each KAW mode over one wave period. The figure shows that the modes with $\beta_{i} \gtrsim 0.5$ have nearly the same normalized damping rate at all scales, which is very different from the dampings of the modes at $\beta_{i}=0.01$ and $\beta_{i}=0.1$. These latters are shown to undergo a strong damping (defined when $2 \pi \gamma / \omega_{r} \sim 1$ ) at respectively $k \rho_{i} \sim 3$ and $k \rho_{i} \sim 10$. This may explain the absence of correlation between the slopes and $\beta_{i}$ reported in Fig. 12 since most of the observations reported here have $\beta_{i} \gtrsim 0.5$. Larger samples of SW data would allow one to obtain lower values of $\beta_{i}$ and to test the scenario proposed here. Other explanations are also possible such as the presence of non-local effects (due to large scales shear flows) that would overcome the strong damping at low $\beta_{i}$ and sustain the cascade down to the electron scales as suggested in Howes et al. (2011c). Another explanation is that KAW scenario discussed here may be irrelevant to interpret the present observations. In any case, the origin of the large spread of the slopes above $f_{\rho_{e}}$ reported here and in magnetosheath turbulence (Huang et al. 2013) (as compared to those in the dispersive range) remains unclear and suggests the lack of universality of the turbulence at these small scales. The scaling of the
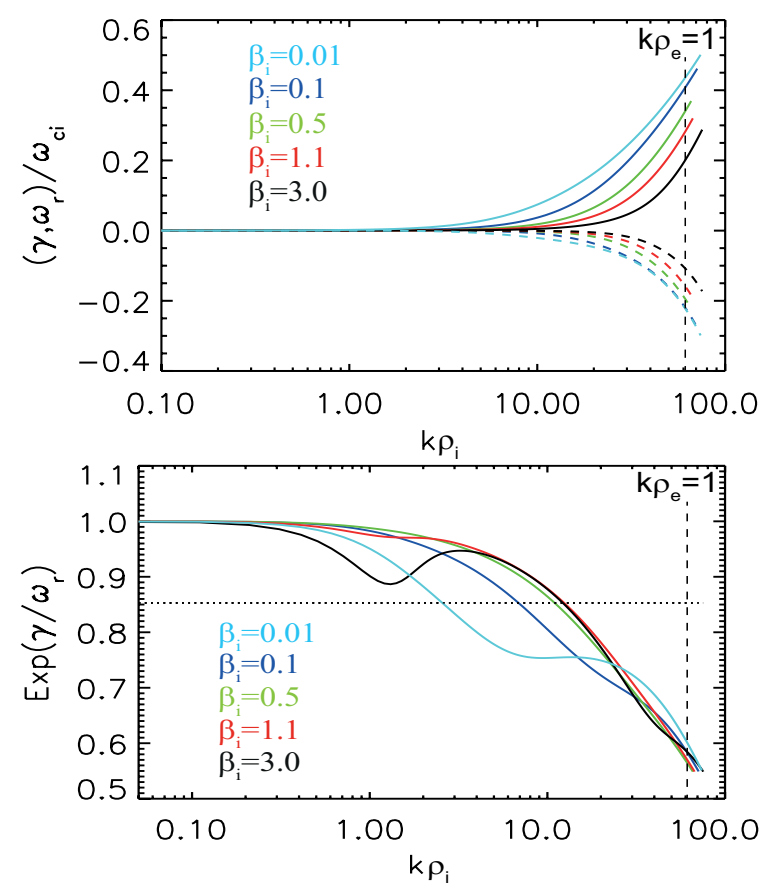

FIG. 13.- Top: KAWs dispersion relations and dampings rates normalized to the proton gyrofrequency for $\theta_{\mathbf{k B}}=89.99^{\circ}$ and the given values of $\beta_{i}$. Bottom: the damping rates of the same modes normalized to one wave period.

spectra at sub-electron scales may depend on local electron plasma instabilities. Such dependence was indeed found between the slopes of the spectra at sub-ion scales and the local ion plasma instabilities (Bale et al. 2009). Proving the existence of the same dependance at subelectron scale requires having high time resolution measurements (a few ms) of electron distribution functions. Such measurements are not available with the current space missions. The NASA/MMS mission is expected to meet part of these requirements.

\section{CONCLUSIONS}

We have surveyed more than ten years of the Cluster burst mode SCM data in the SW and focused on the magnetic energy spectra below the ion gyroscale $\rho_{i}$. We discussed several instrumental caveats that potentially may influence the estimation of the scaling of the spectra at electron scales. We showed in particular that high SNRs are needed in order to determine properly the scaling of the energy spectra. Once all the caveats were considered we found a large number of spectra having clear spectral breaks near the Taylor-shifted electron gyroscale $f_{\rho_{e}}$. A double-power-law model has been adopted to fit the spectra. The distribution of the slopes below the spectral break $f_{b}$ was found to be narrow and centered around $\sim-2.8$, while that above the $f_{b}$ was found wider and centered near the value $\sim-4$. The peak of the distribution of the slopes below $f_{b}$ is consistent with similar observations of SW and magnetosheath turbulence (Alexandrova et al. 2012, Huang et al. 2013) and with most of the existing theoretical or numerical predictions. The spectra above $f_{b}$ are however shallower than those reported in magnetosheath turbulence. A possible explanation of that discrepancy is the difference in the SNR between the SW and the magnetosheath. A moderate correlation was indeed found between small values of the 
slopes, typically $|\alpha|<4$, with lower values of SNR. The reported wide distribution of the slopes combined with earlier observations that showed exponential-like scaling indicate that the physics of the electron scales is possibly not universal and might be controlled by several plasma parameters. Based on the assumption that KAW turbulence dominates at electron scales and on the fact that the linear damping of the KAW modes (from the VlasovMaxwell equations) strongly depends on $\beta_{i}$, an attempt to find a correlation between the observed slopes and the plasma $\beta_{i}$ was carried-out, but the result was not conclusive.

The Cluster-Search Coil data allow for unprecedented in-depth studies of electron scales turbulence in the SW. However, as shown in this study, magnetic field data with higher SNR is now needed to fully and unambiguously characterize the turbulence cascade and dissipation at electron scales in the SW. Besides that one needs furthermore to have accurate measurements of electric field fluctuations and high time resolution of ions and electrons distribution functions, which are not available on current space missions. A new mission, TOR, is currently being designed to fulfill these requirements. TOR should allow in the upcoming years to fully resolve the problem of turbulence cascade and dissipation at electron scales in the SW.

This work is part of the project THESOW funded by L'Agence Nationale de la Recherche (ANR, France). J. De Patoul is funded through the ANR grant. The FGM and CIS data come from the CAA (ESA) and AMDA (CDPP, IRAP, France). FS thanks O. Gurcan for fruitful discussions.

\section{REFERENCES}

Alexandrova, O., Saur, J., Lacombe, C., Mangeney, A., Mitchell, J., Schwartz, S. J., \& Robert, P. 2009, Phys. Rev. Lett., 103, 165003

Alexandrova, Lacombe, C., Mangeney, A., Grappin, R., \& Maksimovic, M. 2012, ApJ, 760, 121

Bale, S. D. , Kellogg, P. J. , Mozer, F. S. , Horbury, T. S. , \& Rème, H. 2005, Phys. Rev. Lett., 94, 215002

Bale, S. D. , Kasper, J. C., Howes, G. G., Quataert, E., Salem, C., \& Sundkvist, D. 2009, Phys. Rev. Lett., 103, 211101

Biskamp, D., Schwarz, E., Zeiler, A, Celani, A., \& Drake, J. F. 1999, Phys. Plasmas, 6, 751

Boldyrev, S., \& Perez, J. C., 2012, ApJ, 758, L44

Bourouaine, S., Alexandrova, O., Marsch, E., \& Maksimovic, M. 2012, ApJ, 749

Camporeale, E., \& Burgess., D. 2011, ApJ, 730, 114

Chang, O., Gary, S. P., Wang, J 2011, Geophys. Res. Lett., 38, L22102, doi:10.1029/2011GL049827

Chen, C. H. K. , Horbury,T. S., Schekochihin, A. A., Wicks, R. T., Alexandrova, O., \& Mitchell, J. 2010, Phys. Rev. Lett., 104, 255002

Cornilleau-Wehrlin, N. et al. 2003, Ann. Geophys. 21, 437-456

Gary, S. P. \& Smith, C. W. 2009, J. Geophys. Res., 114, A12105

Gurcan, O. D., X. Garbet, P. Hennequin, P. H. Diamond, A. Casati,\& G. L. Falchetto, Phys. Rev. Lett., 102, 255002.

Hasegawa, A., T. Imamura, K. Mima,\& T. Taniuti, JPSJ, 43, 255002.

Kiyani, K. H., Chapman, C., Khotyaintsev, Yu.V., Dunlop, M.W., \& Sahraoui, F. 2009, Phys. Rev. Lett., 103, 075006

Kiyani, K. H., Chapman, C., Sahraoui, F., Hnat, B., Fauvarque, O., \& Khotyaintsev, Yu.V., 2013, ApJ, 763,10

Leamon, R. J., Smith, C. W., \& Ness, N. F. 1999, J. Geophys. Res., 104, 22331

Meyrand, R., \& Galtier, S. 2010, ApJ, 721, 1421-1424

Meyrand, R., \& Galtier, S. 2012, Phys. Rev. Lett., 109, 194501
Mazelle, C., Private communication

Howes, G., 2009, Nonlin. Processes Geophys., 16, 219

Howes, G., TenBarge, J. M., Dorland, W., Quataert, E., Schekochihin, A., Numata, R., \& Tatsuno, T. 2011, Phys. Rev. Lett., 107, 035004

Howes, G., TenBarge, J. M., Dorland, W. 2011, Phys. Plasmas, 18,102305

Huang, S., Sahraoui, S. 2012, in preparation

Matthaeus, W. H., Servidio, S., \& Dmitruk, P. 2008, Phys. Rev. Lett., 101, 149501

Podesta, J. J., Borovsky, J. E., Gary, \& S.P. 2010, ApJ, 712, 685

Sahraoui, F., Belmont, G., Rezeau, L. 2003b, Phys. Plasmas, 10, 1325

Sahraoui, F. et al., 2004, Ann. Geophys. 22, 2283

Sahraoui, F., Belmont,G., Rezeau, L., Cornilleau-Wehrlin, N.,

Pinçon, J. L. , \& Balogh, A. 2006, Phys. Rev. Lett., 96, 075002

Sahraoui, F., Goldstein, M. L. , Robert, P. , Khotyaintsev, Y. V. 2009, Phys. Rev. Lett., 102, 231102

Sahraoui, F., Goldstein, M. L., Belmont, G., Canu, P., \& Rezeau, L. 2010a, Phys. Rev. Lett., 105, 131101

Sahraoui, F., Goldstein, M. L., Belmont, G., Roux, A., Rezeau, L., Canu, P., Robert, P., Cornilleau-Wehrlin, N., Le Contel, O., Dudok De Wit, T., Pinçon, J.L., \& Kiyani, K. 2010c, Plant. Space Science, 59,585

Sahraoui, F., Belmont, G., \& Goldstein, M. L., ApJ, 748, 100, doi:10.1088/0004-637X/748/2/100

Salem,C. S. , G. G. Howes, D. Sundkvist, S. D. Bale, C. C.

Chaston, C. H. K. Chen, and F. S. Mozer, ApJ, 745, L9, doi:10.1088/2041-8205/745/1/L9

Schekochihin, A., Cowley, S. C., Dorland, W., Hammett, G. W., Howes, G. G., Quataert, E., \& Tatsuno, T. 2009, ApJS, 182, 310 